\title{
Gene expression profiling from endomyocardial biopsy tissue allows distinction between subentities of dilated cardiomyopathy
}

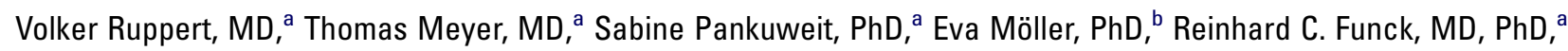
Wolfram Grimm, PhD, and Bernhard Maisch, $\mathrm{MD}^{\mathrm{a}}$ on behalf of the German Heart Failure Network

Supplemental material is available online.
From the Department of Cardiology, University of Marburg, Baldingerstrasse, Marburg, Germany, ${ }^{\mathrm{a}}$ and SIRS-Lab $\mathrm{GmbH}$, Jena, Germany. ${ }^{\mathrm{b}}$

The research on this subject is in part funded by grants from the Deutsche Forschungsgemeinschaft to T. Meyer. V. Ruppert is supported by the BMBF German Heart Failure Network.

Received for publication Dec 17, 2007; revisions received Feb 13, 2008; accepted for publication March 13, 2008.

Address for reprints: Prof Thomas Meyer, Department of Cardiology, University of Marburg, Baldingerstrasse 1,35043 Marburg/Lahn, Germany (E-mail: meyert@med.uni-marburg. de).

J Thorac Cardiovasc Surg 2008;136:360-9 $0022-5223 / \$ 34.00$

Copyright $\odot 2008$ by The American Association for Thoracic Surgery

doi:10.1016/j.jtcvs.2008.03.016
Objective: Expression profile analysis using endomyocardial biopsy specimens from patients with cardiomyopathies promises to improve the differential diagnosis of heart failure.

Methods: In this study, left ventricular endomyocardial biopsy specimens were obtained from 50 patients and histopathologically classified according to the World Heart Federation Task Force criteria as having dilated cardiomyopathy $(\mathrm{n}=17)$, inflammatory cardiomyopathy $(\mathrm{n}=11)$, myocarditis $(\mathrm{n}=15)$, or pericarditis $(\mathrm{n}=7)$. Microarrays were performed by hybridization of synthesized complementary DNA against a Lab-Arraytor60-combi microarray (SIRS-Lab, Jena, Switzerland). Differentially expressed genes were clustered hierarchically according to their variation in hybridization signals.

Results: In samples from patients with dilated cardiomyopathy, two different types of gene expression profiles were distinguishable. One pattern was unique for dilated cardiomyopathy and inflammatory cardiomyopathy, respectively, and the other more closely resembled that seen in samples from inflammatory heart disease. Additionally, we confirmed the microarray data by showing that dilated cardiomyopathy is associated with a reduced myocardial toll-like receptor 9 expression that resulted from progressive loss of functional cardiomyocytes. Taken together, our data demonstrate the utility and validity of microarrays from endomyocardial biopsy specimens in detecting subentities of dilated cardiomyopathy that do not differ histopathologically, but transcriptionally, from each other. The gene expression profile observed in one subgroup of patients with dilated cardiomyopathy is indicative of ongoing immune activation, albeit infiltrating immunocompetent cells were not detected histopathologically.

Conclusion: Thus, our transcriptional data indicate that dilated cardiomyopathy constitutes a heterogeneous disease with an broad overlap to inflammatory heart disease.

$\mathrm{H}$ eart transplantation is the sole surgical option for selected patients with severe cardiomyopathy who do not respond to drug therapy. Accurate clinical assessment is obligatory to decide which patients will benefit from heart transplantation. Several studies have been carried out to determine unfavorable prognoses and to provide an early indication for cardiac transplantation. Despite recent advances in our pathophysiologic knowledge of end-stage heart disease, the differential diagnosis of nonischemic cardiomyopathies still remains a challenge for the clinician, particularly in early stages of the disease. ${ }^{1,2}$

Dilated cardiomyopathy (DCM), a leading cause of heart failure and heart transplantation, is characterized by dilatation and impaired contraction of the left or both ventricles; it may be idiopathic, familial/genetic, viral, and/or immune. Various pathogenic human viruses have been identified as causative agents for myocardial damage, in particular parvovirus B19, enteroviruses (especially Coxsackie B virus), adenoviruses, and herpesviruses including cytomegalovirus. ${ }^{3-6}$ Replication of viral genomes resulting in 


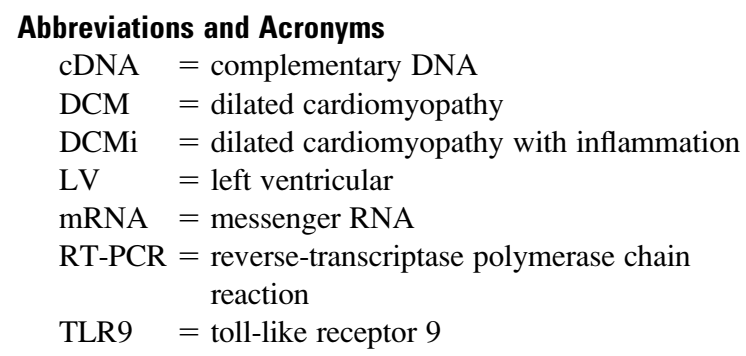

specific epitopes to be tested is restricted depending on the avidity and availability of the corresponding antibodies. Recent advances in microarray technology allow measuring the expression of thousands of genes simultaneously in a defined small tissue sample. ${ }^{12-14}$ Thus, we asked whether microchip technology is principally suitable to distinguish myocarditis from other heart diseases in a diagnostic setting. In particular, we were interested to determine whether gene expression profiling from endomyocardial biopsy tissue adds further information for the identification of patients with cardiomyopathies.

\section{Patients and Methods}

\section{Patient Selection and Diagnosis of Heart Disease}

Patients with signs of heart failure, who had been referred to our hospital for further diagnostic investigation because of suspected inflammatory heart disease, were enrolled in our study. The patients usually had a history of recent onset of cardiac arrhythmias, undefined electrocardiographic abnormalities, and atypical chest pain suggesting nonischemic cardiomyopathy and/or myocardial inflammation. Inclusion criteria for participating in the study were a histologically proven diagnosis of one of the following four cardiac diagnoses: pericarditis, myocarditis, and dilated cardiomyopathy with or without inflammation (DCM and DCMi, respectively). For all study patients the accurate cardiologic diagnosis was obtained before the microchip assays were performed. Patients with pericarditis usually had a hemodynamically relevant pericardial effusion and/or typical electrocardiographic alterations. Myocarditis was defined as an inflammation of the heart according to the World Heart Federation criteria ( $\geq 14$ lymphocytes and macrophages per square millimeter) independent of the clinical phenotype or the presence of heart failure or ventricular dilatation. ${ }^{15,16}$ DCMi was diagnosed if, in addition to histologic signs of inflammation, impaired left ventricular (LV) function was proven by echocardiography (LV end-diastolic diameter $>55 \mathrm{~mm}, \mathrm{LV}$ end-diastolic volume index $>100 \mathrm{~mL} / \mathrm{m}^{2}$, or $\mathrm{LV}$ ejection fraction $<50 \%) .{ }^{16}$ If no inflammation was detectable, patients with impaired LV function were classified as having DCM.

In each patient, coronary angiography was performed to exclude significant coronary artery disease with a luminal narrowing of major arteries of $50 \%$ or more. For all patients, the prevalence of the parvovirus B19 genome was tested as described before. ${ }^{4}$ Patients with suspected inflammatory heart disease who underwent endomyocardial biopsy for therapeutic and/or diagnostic reasons were asked to participate in our study. If they agreed, written informed consent was obtained. The investigation conforms with the principles outlined in the Declaration of Helsinki. This study protocol was approved by the local ethical review board.

\section{Tissue Collection}

From each patient undergoing cardiac catheterization, multiple LV endomyocardial specimens were obtained with a flexible bioptome. Each tissue specimen was then divided into two parts: one for histopathologic examination and the other for RNA analyses. The biopsy specimens were immediately snap-frozen in liquid nitrogen and banked at $-80^{\circ} \mathrm{C}$ for storage until further use.

\section{Immunohistochemical Stainings}

Frozen sections of the banked endomyocardial tissue samples were cut $(5 \mu \mathrm{m})$ and stained immunohistochemically for the detection of has ameliorated the early detection of inflammatory tissue reactions in the heart. ${ }^{4,6,9-11}$ However, the number of cardiac- 
infiltrating cells. Monoclonal antibodies against CD3, CD4, and CD11c, all obtained from Dako Diagnostics Ltd (Copenhagen, Denmark), were used in this study. Avidin-biotin double sandwich technique (Vectastain Elite ABC Kit; Vector Laboratories, Inc, Burlingame, Calif) was used in combination with a monoclonal antibody against the endothelial antigen EN 4 (Sanbio BV, AmUden, The Netherlands) to distinguish infiltrating lymphocytes (red staining) adjacent to myocytes from those located nearby or inside intramyocardial vessels and capillaries (blue staining). Toll-like receptor 9 (TLR9) was stained with a rabbit polyclonal antibody (H-100) purchased from Santa Cruz Biotechnology (Santa Cruz, Calif). Detection of bound immunoglobulins was achieved with the ABC method employing biotinylated secondary antibodies and avidinhorseradish peroxidase complexes (Vectastain Elite ABC Kit from Vector Laboratories). Diaminobenzidine producing a brown reaction product was used as a substrate for visualization of the enzyme reaction. Finally, the sections were counterstained with Mayer's hematoxylin.

\section{RNA Isolation, Complementary DNA Synthesis and Microarray Hybridization}

Total RNA was extracted from endomyocardial biopsy specimens or autoptic heart tissue using the Qiagen RNeasy Kit and eluted in a volume of $30 \mu \mathrm{L}$ of sterile water as recommended by the manufacturer. RNA concentrations were determined spectrometrically at a wavelength of $260 \mathrm{~nm}$. Because of minimal total RNA in the samples, complementary DNA (cDNA) synthesis was performed by the BD Super SMART cDNA synthesis technology (BD Biosciences, Franklin Lakes, NJ) according to the manufacturer's instructions. Reverse transcription was in the presence of aminoallyl-dUTP and cDNA was labeled by the use of the Alexa Fluor 647 system (Invitrogen, Carlsbad, Calif). Alexa Fluor 647-labeled cDNA was cohybridized with pooled Alexa Fluor 555-labeled cDNA obtained from 6 autoptic hearts from patients who died of noncardiac diseases and served as control samples. Synthesis of cDNA included a DNase I treatment (Promega Corporation, Madison, Wis) to prevent cellular DNA contamination in the polymerase chain reaction (PCR). Each RNA pair (sample vs reference) was hybridized against a Lab-Arraytor60-combi microarray, comprising 578 probes for 556 human genes involved in inflammatory processes and 22 positive/negative controls (SIRS-Lab, Jena, Switzerland). After incubation in a formamide-based hybridization buffer for 10 hours at $42^{\circ} \mathrm{C}$, microarrays were washed and dried. Hybridization signal intensities were measured with a GenePix scanner (Axon Instruments, division of Molecular Dynamics, Sunnyvale, Calif).

\section{Microarray Data Pre-processing}

Data pre-processing included the following 4 steps: (1) spot detection and quantification, (2) spot flagging according to the defined signal-to-noise threshold value, (3) correction of systematic bias including the normalization and the variance-stabilized transformation of raw signals, and (4) averaging of printing replicates. For the first 2 steps, the GenePix Analysis Software was used. The raw expression signals for each spot were quantified as the median spot intensity in the red and green channel, respectively. The spots were flagged corresponding to the settings of the GenePix Software. For the third step the approach of Huber and colleagues ${ }^{17}$ was used and the additive and multiplicative errors were estimated. The normalized signals were transformed by the arcsinh function. Per gene, replicates with the highest flag value were selected and the corresponding signal intensities were averaged.

\section{Statistical Data Analysis}

In the analysis, the gene expression profiles of 50 patients with sufficient hybridization quality were included. The statistical analysis was performed for 481 reliable genes, that is, genes with detectable signal intensity and sufficient variation throughout the experiment. Missing values were imputed employing the k-nearest neighbor algorithm with $\mathrm{k}=15 .^{18} \mathrm{~A}$ vector of $p$ values was obtained from a multivariate permutation test based on 1-way analysis of variance statistics, where the effect of the disease type (DCM, DCMi, myocarditis, and pericarditis) was investigated for each gene. Genes with statistically significant differences were identified by tuning the $q$ value, thus controlling the positive false discovery rate. ${ }^{19}$ The corresponding approach also allowed for estimation of the proportion of differentially expressed genes. The gene expression patterns of selected genes were ordered by hierarchical cluster algorithm with Euclidean distance and averaged linkage method.

\section{Reverse-transcriptase PCR (RT-PCR)}

Verification of the microarray results was confirmed by real-time PCR using the SYBR-Green method (Invitrogen). Gene-specific primers were designed using Primer 3 software (Applied Biosystems, Foster City, Calif) to amplify fragments of about 200 base pairs in length. The reverse-transcriptase (RT) PCR reactions were carried out in a total volume of $25 \mu \mathrm{L}$, containing $25 \mathrm{ng}$ messenger RNA (mRNA), $7.5 \mu \mathrm{mol} / \mathrm{L}$ of each specific primer pair, and $2.5 \mu \mathrm{L}$ of SYBR Green. The following protocol was applied: reverse transcription at $50^{\circ} \mathrm{C}$ for 30 minutes followed by a denaturation step at $95^{\circ} \mathrm{C} 15$ minutes, and 40 cycles of denaturation at $95^{\circ} \mathrm{C}$ for $45 \mathrm{sec}-$ onds, annealing at $60^{\circ} \mathrm{C}$ for 45 seconds, and extension at $72^{\circ} \mathrm{C}$ for 45 seconds. A melting curve analysis was run after final amplification via a temperature gradient from $55^{\circ} \mathrm{C}$ to $94^{\circ} \mathrm{C}$ in $0.5^{\circ} \mathrm{C}$ increment steps measuring fluorescence at each temperature for a period of 10 seconds. All reactions were carried out in at least duplicate for each sample. The relative expression of a transcript was calculated as the ratio between the specific transcript level and the level of gapdh as determined for each sample. Using the Bio-Rad iQ-iCycler system software (Bio-Rad Laboratories, Hercules, Calif), the threshold (Ct) at which the cycle numbers were measured was adjusted to areas of exponential amplification of the traces. The $\Delta \Delta$-method was used to determine comparative relative expression levels by applying the formula $2^{-(\Delta \mathrm{Ct} \text { target }-\Delta \mathrm{Ct} \text { reference sample })}$, as described previously. ${ }^{20}$

\section{Results}

\section{Characterization of the Study Population}

The total study population included 50 patients. Among them, DCM was diagnosed in 17 study participants and DCMi in 11 subjects. The diagnosis of myocarditis was confirmed in 15 patients. A fourth group consisting of 7 subjects with pericardial effusion was included in the study, in which endomyocardial biopsy had revealed no pathologic signs on histologic examination. This group of patients was therefore diagnosed to have pericarditis. With the exception of the latter group, the majority of all study participants in each group were men (DCM, 89.9\%; DCMi, 90.0\%; and myocarditis, 
TABLE 1. Patient characteristics

\begin{tabular}{lllll}
\hline & \multicolumn{1}{c}{ DCM } & \multicolumn{1}{c}{ DCMi } & Myocarditis & \multicolumn{1}{c}{ Pericarditis } \\
\hline Age (y) & $48.8 \pm 14.6$ & $45.8 \pm 9.6$ & $41.7 \pm 19.7$ & $50.6 \pm 21.7$ \\
Sex (F/M) & $2 / 15$ & $1 / 8$ & $3 / 12$ & $4 / 3$ \\
EF (\%) & $27.2 \pm 10.6^{*}$ & $27.8 \pm 10.0^{*}$ & $69.1 \pm 14.8$ & $65.1 \pm 22.5$ \\
LVEDD (mm) & $67.3 \pm 6.9^{*}$ & $64.5 \pm 7.0^{*}$ & $50.2 \pm 5.3$ & $45.1 \pm 4.2$ \\
Parvovirus B19 (pos./neg.) & $4 / 13$ & $4 / 5$ & $7 / 8$ & $3 / 4$ \\
\hline
\end{tabular}

EF, Ejection fraction; $L V E D D$, left ventricular end-diastolic diameter; pos./neg., positive/negative. ${ }^{*} P<.05$; difference between patient groups having myocarditis or pericarditis.

82.3\%). As expected, the echocardiographically measured LV end-diastolic diameter was significantly elevated in both DCM and DCMi patients $(67.3 \pm 6.9 \mathrm{~mm}$ and $64.5 \pm$ $7.0 \mathrm{~mm}$, respectively) as compared with patients with myocarditis or pericarditis $(50.2 \pm 5.3 \mathrm{~mm}$ and $45.1 \pm$ $4.2 \mathrm{~mm} ; P<.05)$. Similarly, LV ejection fraction differed significantly between DCM and DCMi patients on the one hand $(27.2 \% \pm 10.6 \%$ and $27.8 \% \pm 9.6 \%$, respectively $)$ and patients with myocarditis or pericarditis on the other $(69.1 \% \pm 14.8 \%$ and $65.1 \% \pm 22.5 \% ; P<.05)$. Further details on the study population are presented in Table 1.

\section{Immunohistochemical Detection of}

\section{Immunocompetent Cells in Biopsy Tissue}

Immunohistochemistry was performed in biopsy samples to detect immunocompetent cells in the myocardium, thereby distinguishing between different entities of heart diseases. Figure 1 demonstrates the presence of CD3-positive T cells in mononuclear infiltrates in patients with myocarditis, whereas in patients with DCM and pericarditis staining with anti-CD3 antibody was usually negative (Figure 1, A). In endomyocardial biopsy tissue from patients with DCMi, CD3-positive $\mathrm{T}$ cells only occasionally were detected scattered throughout the section. Similarly, CD4-positive cells were detected in specimens from inflammatory heart disease and infrequently in patients with DCMi but were generally absent in DCM and pericarditis (Figure 1, $B$ and $C$ ). Dendritic cells expressing CD11c were most often detected in inflammatory infiltrates from patients with myocarditis (Figure 1, $D)$. However, dendritic cells were also detected but less frequently in tissue specimens from the remaining patient groups.

\section{Differential Gene Expression in Different Heart \\ Diseases}

Next, we performed microarrays to explore putative differences in the expression profile of genes involved in inflammatory reactions among the four entities of heart disease. A list of genes that showed differential expression between the patient groups was generated by ranking the genes according to the
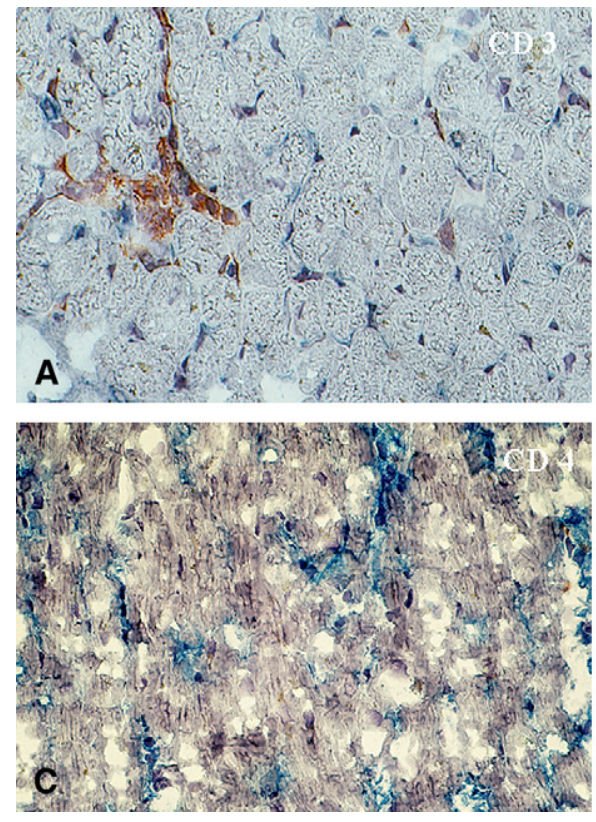
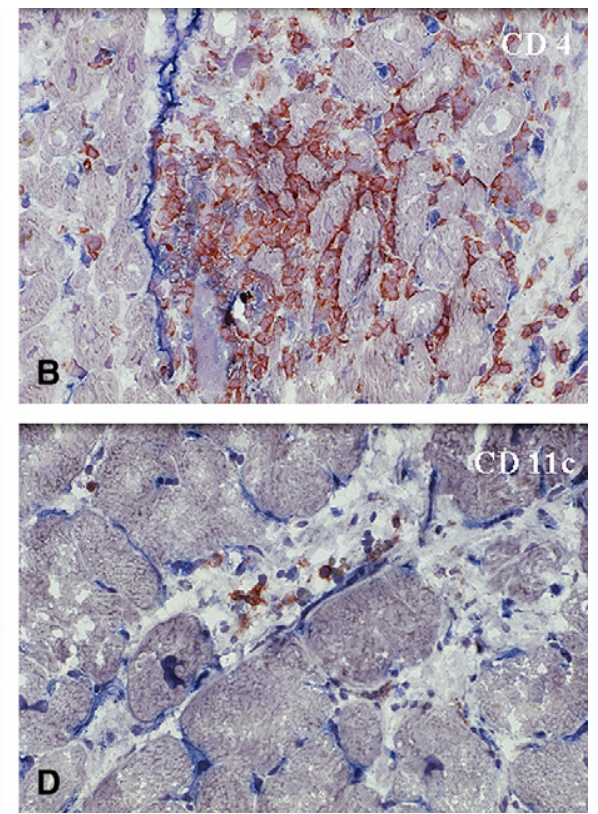

Figure 1. Immunohistochemical detection of infiltrating immune cells in endomyocardial biopsy samples. Shown are the localization of CD3- (A) and CD4positive $T$ cells ( $B$ and $C$ ) in patients with myocarditis (A and B) and DCM (C), respectively, as well as CD11c-positive dendritic cells in a patient with myocarditis (D). Double staining using a monoclonal antibody against endothelial antigen EN 4 was applied to distinguish infiltrating lymphocytes (red staining) from intramyocardial vessels and capillaries (blue staining). Note the absence of a positive CD4 immunostaining in heart tissue obtained from a DCM patient (C). 
relative median expression. We found differences in the gene expression between the patient groups for approximately $45 \%$ of genes tested. A set of 42 genes with a $q$ value of less than 17 (corresponding to a $p$ value of less than.026) was considered as being significantly changed (thus 7 false positive decisions were accepted). Data corresponding to the selected genes are summarized in Supplemental Table E1 and Figures 2 and 5.

The mean expression of the 6 genes with least $q$ values (cflar, c3, serca2, serping, cis, and galc) was higher for the patient groups with myocarditis and pericarditis, respectively, as compared with those with DCMi or DCM. For the majority of the selected genes the expression level was elevated in the patients with myocarditis and pericarditis as compared with study participants with DCM or DCMi. The expression of some genes, for example, fos, nppb, map $3 k$, tnfrsf6, or $p p b p$, was reduced in the group with pericarditis when compared with the remaining patient groups. To better visualize the obtained expression patterns, variations in hybridization signals were ordered by hierarchical clustering. In Figure 2, the transcription patterns are presented by a colored image and the corresponding dendrogram. It can be seen in this figure that with respect to the gene expression pattern, the patient group with DCM encompasses two distinct subgroups. Whereas the gene expression in 7 patients of the DCM group was similar to that observed in myocarditis, the gene expression in the remaining 10 patients corresponded well to that seen in patients with DCM. Interestingly, the former subgroup of patients with DCM, who more likely resembled the expression profile of myocarditis patients, had significantly higher LV shortening fractions $(18.9 \% \pm 6.3 \%$ vs $11.3 \% \pm 3.0 \% ; P=.003$; see Figure 3$)$.

Next, we compared the two DCM subgroups separately with the DCMi patients for their echocardiographically assessed shortening fraction (for DCMi patients; $16.0 \% \pm$ $3.2 \%)$. Statistical significance was achieved only for the subgroup of DCM patients with a unique gene expression $(P=$ .003), but not for those DCM patients with a gene expression pattern resembling that of myocarditis $(P>.05)$. No association was seen between parvovirus B19 positivity and gene expression profiling.

\section{Validation of Differentially Expressed Genes Using RT-PCR}

The differential gene expression observed in the microarrays was verified by two independent measurement methods. First, we performed real-time RT-PCR from the same set of tissue samples and compared these results with the findings from the microarrays. On the basis of biologic considerations and statistical significance, two genes were selected for RTPCR measurements: serca and tlr 9 . The sarcoplasmic reticulum $\mathrm{Ca}^{2+}$-ATPase Serca was included in the microarray and chosen for RT-PCR validation, because it is widely known that Serca expression is down-regulated in the hearts of patients with DCM. ${ }^{21}$ The second gene included for validation of the microarray results by quantitative RT-PCR was $t r r$. Expression of gapdh was not significantly changed between the samples and therefore gapdh served as a control for normalization of the RT-PCR measurements.

Our RT-PCR results confirmed that serca expression in DCM and DCMi patients was down-regulated as compared with that in patients with pericarditis or myocarditis (Figure 4, B). The decreased serca expression in the former patient groups as measured by RT-PCR correlated well with the results from the microchip experiment. Similarly, expression of $t r r 9$ coding for TLR9 was significantly reduced in DCM patients as judged by both RT-PCR and microarrays (Figure $4, A$ ). The expression level of $t l r 9$ was intermediate in DCMi patients and high in patients with acute inflammatory heart disease. Thus, our data showed that the RT-PCR results generally paralleled those seen by microarray analysis.

\section{Expression of TLR9 in Endomyocardial Biopsy Tissue}

Next, we tested whether immunohistochemical stainings confirmed the expression data obtained from the microarrays (Figure 5). Since microarray and RT-PCR techniques both revealed down-regulation of $t / r 9$ in DCM and DCMi patients, we asked whether the reduced TLR9 expression was also detectable at the level of protein expression. In immunohistochemical stainings using a specific anti-TLR9 antibody, we confirmed the expression patterns described above at the mRNA level. TLR9 was localized predominantly in the cytosol of cardiomyocytes but was generally absent in noncardiomyoctes. It was found that the staining intensity of TLR9 immunopositivity in biopsy specimens from patients with DCM was significantly lower as compared with the other three disease entities (Figure 5,D). There was a significantly higher expression of TLR9 in tissue samples from myocarditis and pericarditis patients (Figure $5, A$ and $B$ ). Also here TLR9-positive cells were exclusively cardiomyocytes as judged by their characteristic shape and the presence of a contractile apparatus, whereas lymphocytes or antigen-presenting cells comprising inflammatory infiltrates were typically negative. Biopsy samples from patients with DCMi often showed an intermediate staining pattern (Figure 5, C). Taken together, immunohistochemical detection of TLR9 used here as an independent method for assessing gene expression confirmed the differential gene expression pattern between the distinct disease conditions, as had already been revealed before by microarray and RT-PCR techniques.

\section{Discussion}

In this study, we compared the global gene expression profiles from four clinically and histopathologically defined entities of heart disease using a custom-designed DNA microarray technology in an attempt to identify potential molecular markers that facilitate the differential diagnosis of cardiomyopathies. The set of genes tested here for cardiac 

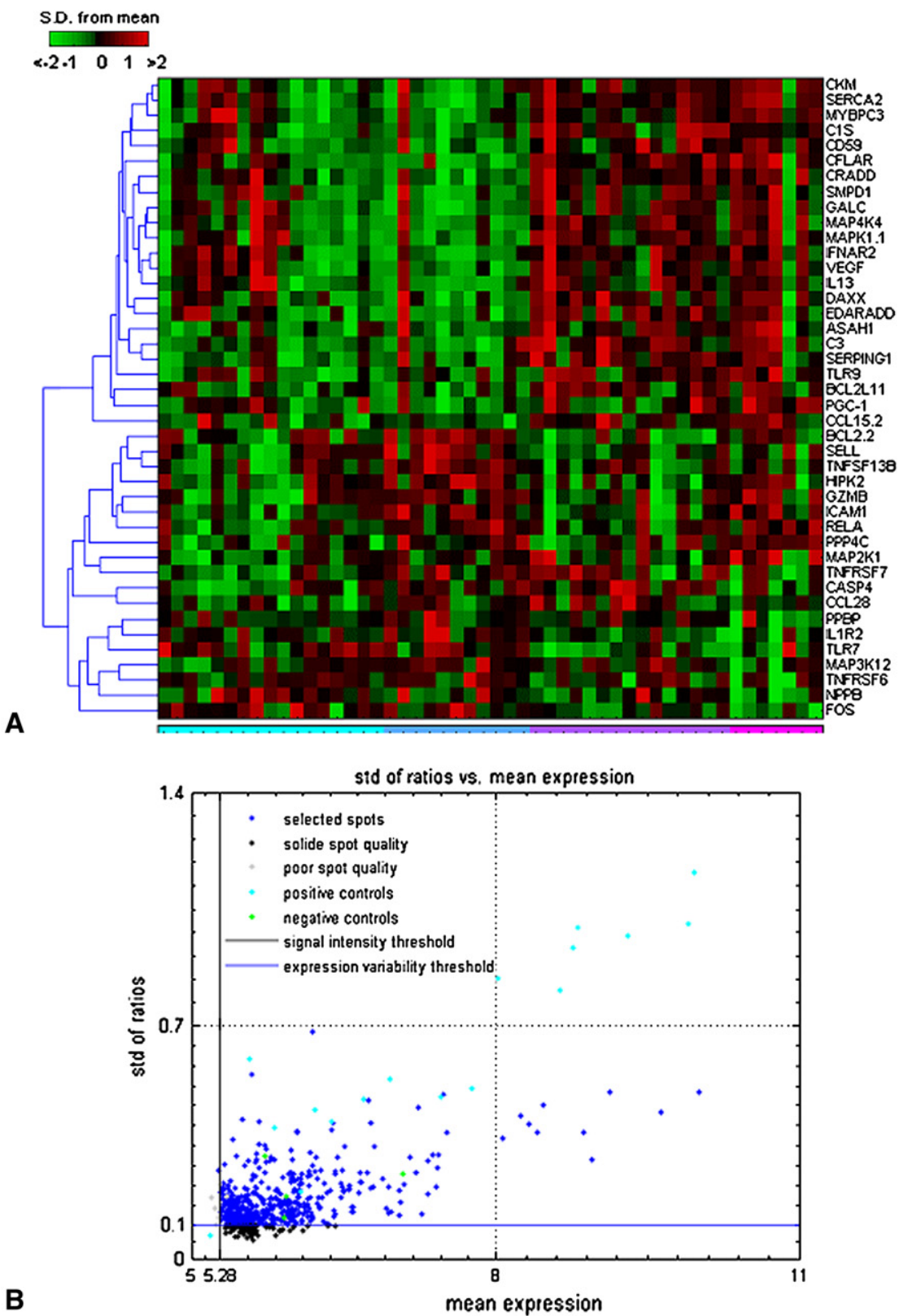

B

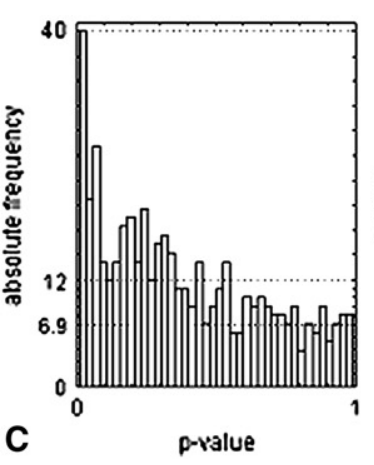

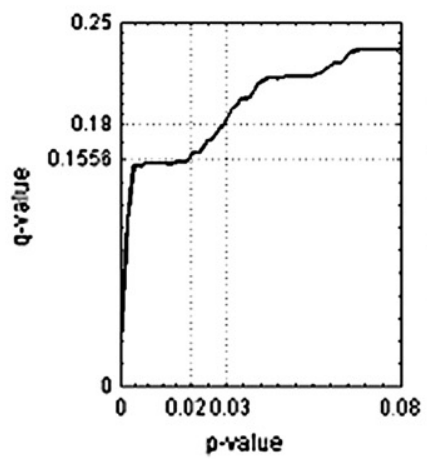

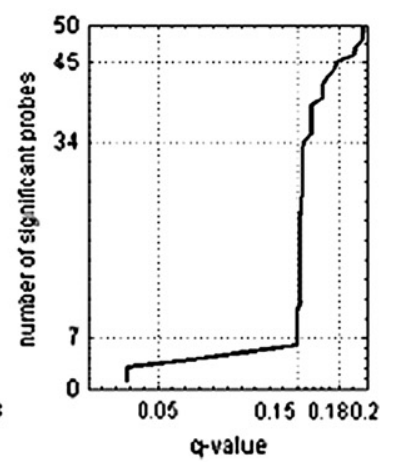

Figure 2. Gene expression characteristics in patients with different clinical diagnoses. From statistical comparison, 42 genes were identified that significantly differed between the patient groups. The gene expression patterns of these genes were ordered by hierarchical cluster algorithm with Euclidian distance and averaged linkage method. Each gene is represented by a single row of colored boxes and each sample by a single column. The associated colors represent variance-normalized expression for individual genes. While the intensity of red color indicates a relative expression greater than the mean, green indicates a decreased expresion level compared with the mean. (B) Distribution of the gene expression throughout the experiment. To minimize the number of false positives, we excluded such genes from the statistical analysis whose mean expression intensity were less than those of any the negative control or whose expression variation throughout the experiment was less than those of the most positive controls. (C) Statistical comparison of the gene expression patterns. Left panel, Histogram of $p$ values obtained from the statistical test. While the line at the height of ho=12 is the histogram expected portion of null $p$ values if none gene was differentially expressed, the line at the height of $h 1=6.9$ is the histogram of the estimated portion of null $p$ values. Middle panel, The $q$ values versus their respective $p$ values. Right panel, The number of genes considered as being differentially expressed versus the respective $q$ values. For a $q$ value closed to 0.17 a particular increase in the curve can be observed. 

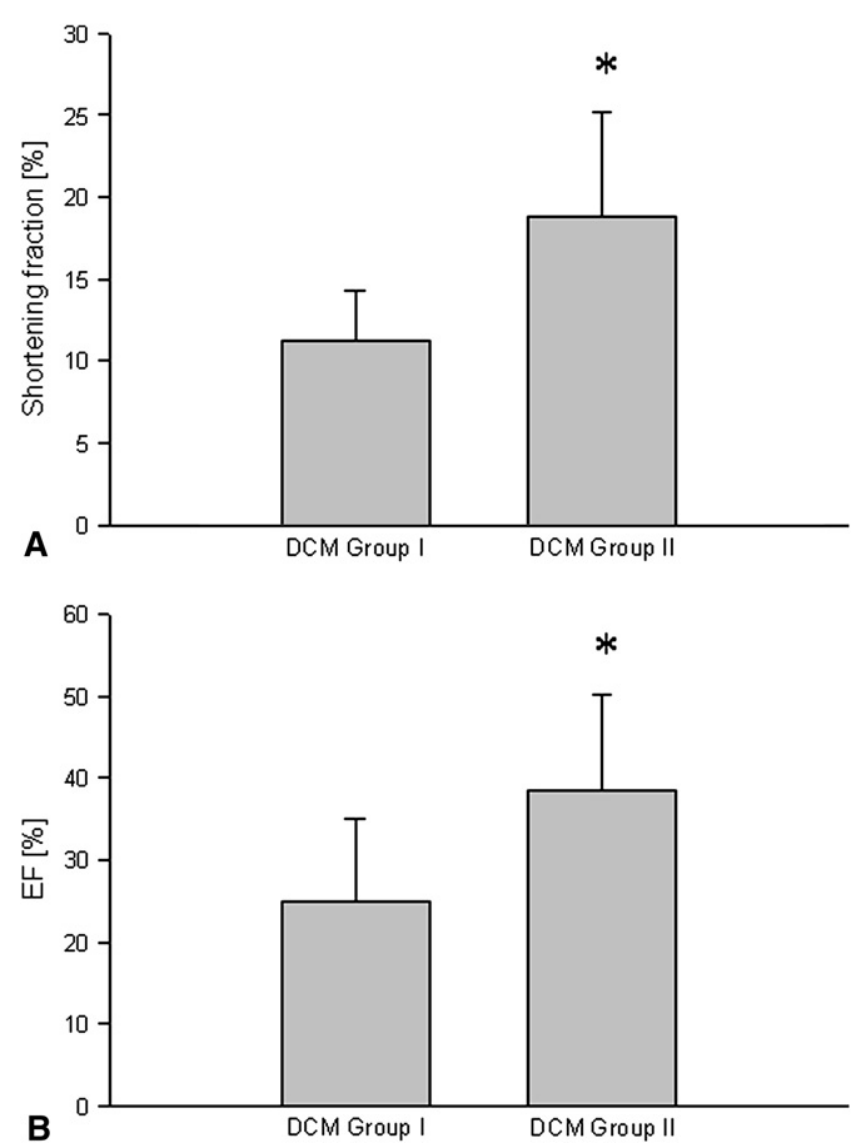

Figure 3. The gene expression profiles between two subgroups of DCM patients differ significantly depending on the severity of impaired LV systolic function. Echocardiographically measured shortening fractions $(A)$ and ejection fractions (EF) (B) are presented separately for both subgroups of DCM patients with distinct gene expression patterns.

expression was selected on the basis of the involvement of the corresponding gene products in executing inflammatory reactions. The data presented demonstrate that hierarchical gene clustering in microarray analysis allows for the identification of distinct gene expression patterns that distinguish different heart diseases from each other. Despite considerable interindividual variability, our microarray results show that in the myocardium of patients with DCM and DCMi, a panel of genes involved in immune regulation is differentially expressed as compared with that in patients with myocarditis or pericarditis. Within the group of DCM patients we observed two different expression patterns for inflammatory markers, which were clearly distinguishable from each other. One gene expression profile resembled more closely that seen also in patients with myocarditis, whereas the other pattern was unique for DCM and DCMi patients. Individuals exhibiting the latter type of gene expression had a significantly reduced LV shortening fraction as compared with those from the former subgroup, indicating that their LV systolic function was more profoundly impaired. These data suggest that DCM patients with a gene expression pattern more closely resembling that of myocarditis may be classified as belonging to an intermediate subgroup that is transcriptionally, but not necessarily histopathologically, distinguishable from subjects with a DCM-specific gene expression profile.

Additionally, our microarray results indicate that there is a substantial overlap in gene expression profiles between the subgroup of DCM patients with only slightly impaired LV systolic function and those diagnosed as DCMi. In the subgroup of DCM patients exhibiting a unique gene expression pattern, the shortening fraction was significantly more reduced as compared with that of patients with a transcription pattern resembling that of myocarditis. Thus, belonging to a transcriptionally defined DCM subgroup seems to be associated with a reduced systolic function of the left ventricle. The different myocardial gene expression profiles seen in both DCM subgroups appear to more likely reflect the severity of LV impairment than the presence of a still ongoing immune reaction, which is undetectable in the diverse immunohistochemical techniques applied here for the detection of infiltrating cells.

The expression levels of the selected genes were generally more decreased in DCM and DCMi patients as compared with those diagnosed as either myocarditis or pericarditis. This finding was not unexpected inasmuch as the genes selected for the microarrays were chosen with respect to their engagement in inflammatory processes. However, it is unclear whether the reduced activation level found here for numerous genes simply reflects the nosologic heterogeneity and clinical severity of the disease or whether it indicates a more profound alteration in transcriptional activity that accompanies the phenotypic dedifferentiation process of cardiomyocytes. Nevertheless, our microarray results allow DCM patients to be divided into two distinct subgroups that differ considerably with respect to the transcriptional activation of inflammatory genes. Thus, microarrays obtained from endomyocardial biopsy tissue add further information for the subclassification of DCM patients that are not covered by conventional methods of gene expression measurements.

Additionally, we tested the microarray technique for screening novel markers whose expression is specifically changed in DCM. Recently, this approach has been successfully applied in numerous studies. ${ }^{14,22-31}$ Here we focused on a gene product that has not been related to the pathogenesis of DCM so far, but whose expression was gradually reduced as determined in our microarrays. The gene coding for TLR9 was identified as such a marker that is significantly down-regulated in DCM patients. Interestingly, our RT-PCR results confirmed that the induction of the $t l r 9$ gene is critically impaired in DCM and DCMi patients, whereas significantly higher expression levels were measured in myocarditis and pericarditis patients. Expression of TLR9 was restricted to 

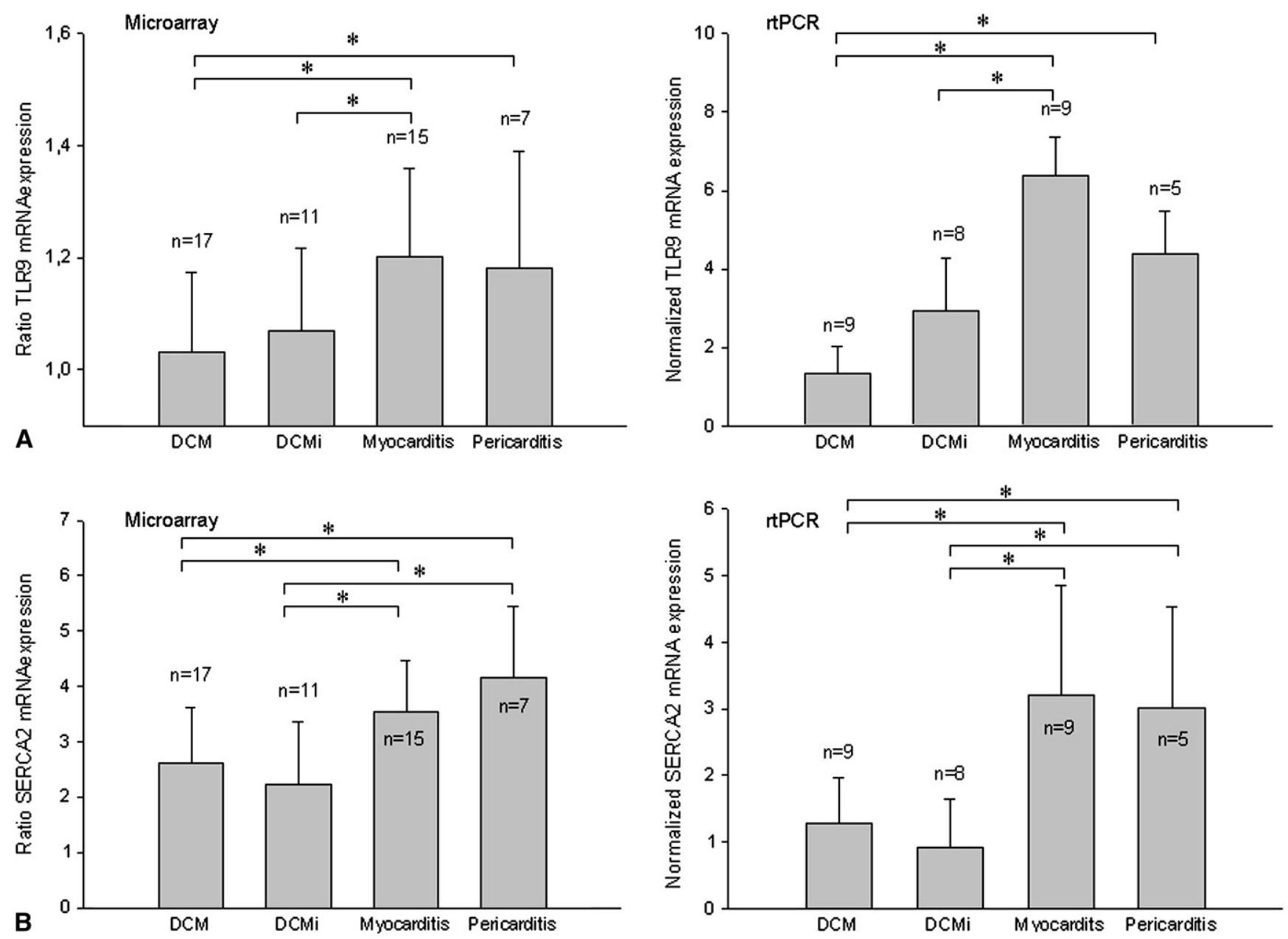

Figure 4. Comparison of expression data between microarrays and RT-PCR measurements. Shown are the microarray (left) and RT-PCR results (right) for the expression of tIr9 coding for toll-like receptor 9 (A) and serca (B) in patients grouped with respect to the underlying heart disease. Note that serca and $t / r 9$ mRNA are both down-regulated in DCM and DCMi as compared with patients with myocarditis or pericarditis and that these findings were independent of the particular technique used.

differentiated cardiomyocytes as observed immunohistochemically, whereas nonmyocytes usually expressed much lower, if any, immunodetectable TLR9. With increasing amounts of fibrotic components, the signal intensity in the anti-TLR9 stained tissue specimens was critically decreased, as was observed in DCM patients. Thus, three independent techniques demonstrated down-regulation of the $\operatorname{tr} 9$ gene as was first revealed in the microarray analyses and later confirmed both at the mRNA and protein level. Members of the toll-like receptor family such as TLR9 have been implicated in the recognition of exogenous and endogenous ligands and activate the nuclear factor $\kappa \mathrm{B}$ pathway. ${ }^{32}$ The high expression level of TLR9 in morphologically intact cardiomyocytes suggests a functional role of these cells in the recognition of the cognate ligand, which is unmethylated CpG DNA.
Our data suggest that implementing microarray-based information on gene expression broadens our understanding for the pathogenesis and subclassification of DCM and related diseases. RNA amplification protocols combined with gene expression profiling appear to refine the clinical diagnosis of cardiomyopathies. Novel disease-associated markers may be identified that are suitable for the screening of patients with otherwise unexplained heart disease. Advanced DNA microchip technology promises to identify target genes of potential therapeutic interest that are involved in diverse pathologic pathways such as remodeling and apoptosis. The expression of these genes may then be specifically modulated by gene-targeting approaches that are currently under development.

Taken together, our microarray analysis based on genes involved in immune regulation allows for the distinction 

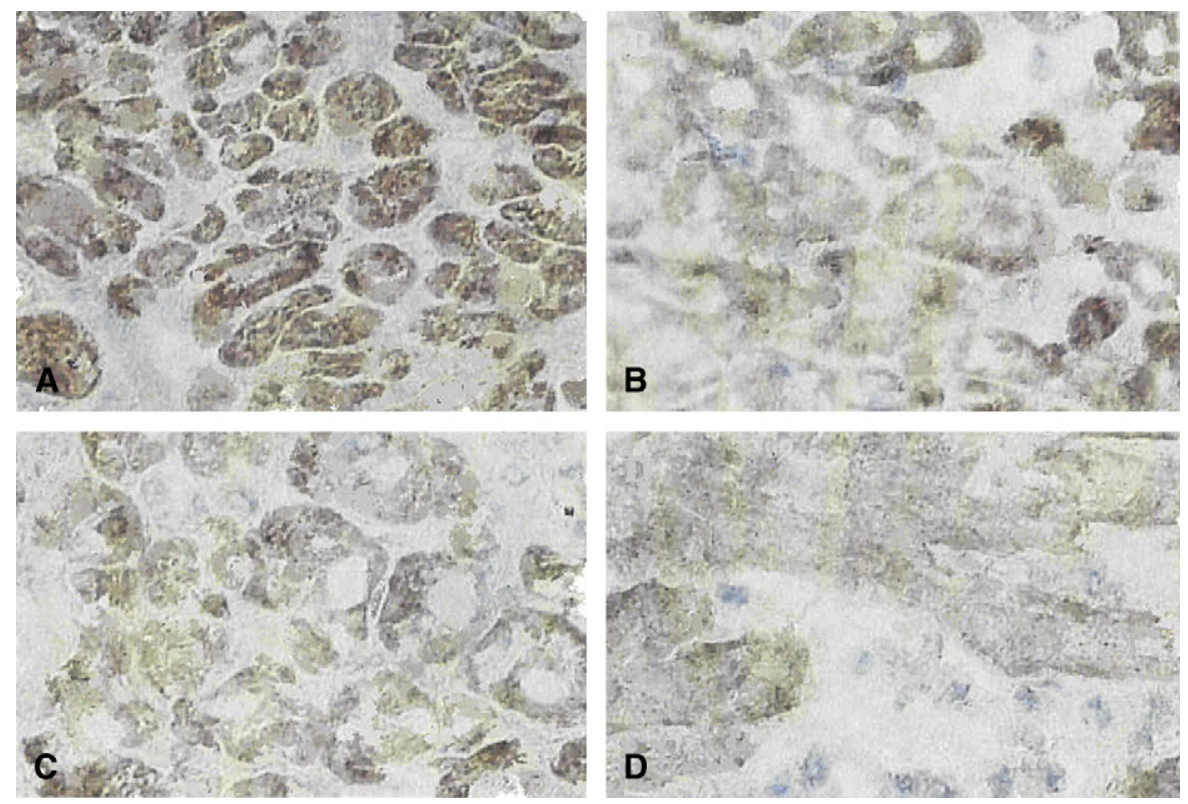

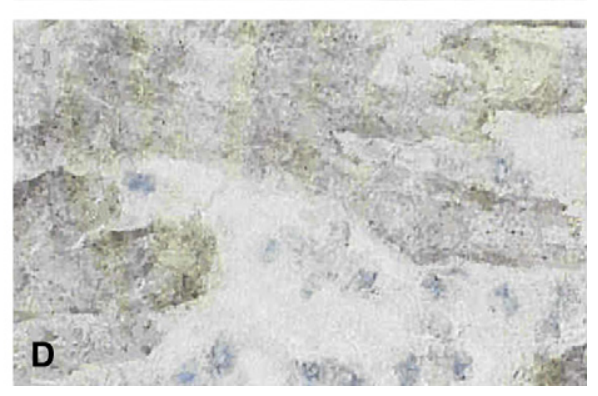

Figure 5. Decreased expression of tolllike receptor 9 (TLR9) in DCM and DCMi patients as shown by immunohistochemistry. Endomyocardial biopsy specimens were stained for the presence of TLR9 in patients diagnosed as myocarditis (A), pericarditis (B), DCMi (C), and DCM (D), respectively. between different entities of DCM. In a subgroup of DCM patients with impaired LV function, we detected evidence of continuous immune activation, as judged by the elevated expression of inflammatory genes. Thus, despite the absence of infiltrating immunocompetent cells, our transcriptional data demonstrate ongoing activation of the immune system in this DCM subgroup. The robustness of the gene expression signatures shown here can be successfully applied to identify novel disease-associated markers, such as TLR9, that are dysregulated in specific cardiac entities.

We gratefully acknowledge the expert support by Markus Bläss and Stefan Rußwurm from SIRC Lab for performing microarray analysis and the excellent technical assistance by Marlies Crombach and Heike Eckhardt from the University of Marburg.

\section{References}

1. Pisani B, Taylor DO, Mason JW. Inflammatory myocardial diseases and cardiomyopathies. Am J Med. 1997;102:459-69.

2. Magnani JW, Dec GW. Myocarditis. Current trends in diagnosis and treatment. Circulation. 2006;113:876-90.

3. Maisch B, Richter A, Kölsch S, Alter P, Funck R, Pankuweit S. Management of patients with suspected (peri-)myocarditis and inflammatory dilated cardiomyopathy. Herz. 2006;31:881-90.

4. Pankuweit S, Moll R, Baandrup U, Portig I, Hufnagel G, Maisch B. Prevalence of the parvovirus B19 genome in endomyocardial biopsy specimens. Hum Pathol. 2003;34:497-503.

5. Kühl U, Pauschinger M, Noutsias M, Seeberg B, Bock T, Lassner D, et al. High prevalence of viral genomes and multiple viral infections in the myocardium of adults with "idiopathic "left ventricular dysfunction. Circulation. 2005;111:887-93.

6. Mahrholdt H, Wagner A, Deluigi CC, Kispert E, Hager S, Meinhardt G, et al. Presentation, patterns of myocardial damage, and clinical couse of viral myocarditis. Circulation. 2006;114:1581-90.

7. Ardehali H, Qasim A, Cappola T, Howard D, Hruban R, Hare JM, et al. Endomyocardial biopsy plays a role in diagnosing patients with unexplained cardiomyopathy. Am Heart J. 2004;147:919-23.
8. Mills RM, Lauer MS. Endomyocardial biopsy: a procedure in search of an indication. Am Heart J. 2004;147:759-60.

9. Aretz HT, Billingham ME, Edwards WD, Factor SM, Fallon JT, Fenoglio JJ, et al. Myocarditis. A histopathologic definition and classification. Am J Cardiovasc Pathol. 1987;1:3-14.

10. Herskowitz A, Campbell S, Deckers J, Kasper EK, Boehmer J, Hadian D, et al. Demographic features and prevalence of idiopathic myocarditis in patients undergoing endomyocardial biopsy. Am J Cardiol. 1993;71:982-6.

11. Zimmermann O, Kochs M, Zwaka TP, Kaya Z, Lepper PM, BienekZiolkowski M, et al. Myocardial biopsy based classification and treatment in patients with dilated cardiomyopathy. Int J Cardiol. 2005; 104:92-100.

12. Cook SA, Rosenzweig A. DNA microarrays. Implications for cardiovascular medicine. Circ Res. 2002;91:559-64.

13. Perrot A, Kabaeva Z, Wenzel K, Osterziel KJ. Gene expression analysis of human tissue from patients with cardiomyopathies: a new tool for guiding therapies in the future? J Card Surg. 2005;20:S17-9.

14. Nanni L, Romualdi C, Maseri A, Lanfranchi G. Differential gene expression profiling in genetic and multifactorial cardiovascular diseases. J Mol Cell Cardiol. 2006;41:934-48.

15. Richardson P, McKenna W, Bristow M, Maisch B, Mautner B, O'Connell J, et al. Report of the 1995 World Health Organization/International Society and Federation of Cardiology Task Force on the Definition and Classification of Cardiomyopathies. Circulation. 1996;93: 841-2.

16. Maisch B, Richter A, Sandmöller A, Portig I, Pankuweit S, BMBFHeart Failure Network. Inflammatory dilated cardiomyopathy. Herz. 2005;30:535-44.

17. Huber W, von Heydebreck A, Sueltmann H, Poustka A, Vingron M. Parameter estimation for the calibration and variance stabilization of microarray data. Stat Appl Genet Mol Biol. 2003;2:Article 3.

18. Troyanskaya O, Cantor M, Sherlock G, Brown P, Hastie T, Tibshirani R, et al. Missing value estimation methods for DNA microarrays. Bioinformatics. 2001;17:520-5.

19. Storey JD, Tibshirani R. Statistical significance for genome-wide studies. Proc Natl Acad Sci U S A. 2003;100:9440-5.

20. Pfaffl MW. A new mathematical model for relative quantification in realtime RT-PCR. Nucleic Acids Res. 2001;29:e45.

21. Isenberg $\mathrm{G}$. How can overexpression of $\mathrm{Na}^{+}, \mathrm{Ca}^{2+}$-exchanger compensate the negative inotropic effects of downregulated SERCA? Cardiovasc Res. 2001;49:1-6. 
22. Sehl PD, Tai JTN, Hillan KJ, Brown LA, Goddard A, Yang R, et al. Application of cDNA Microarrays in determining molecular phenotype in cardiac growth, development, and response to injury. Circulation. 2000;101:1990-9.

23. Yang J, Moravec CS, Sussman MA, DiPaola NR, Fu D, Hawthorn L, et al. Decreased SLIM1 expression and increased gelsolin expression in failing human hearts measured by high-density oligonucleotide arrays. Circulation. 2000;102:3046-52.

24. Barrans JB, Allen PD, Stamatiou D, Dzau VJ, Liew CC. Global gene expression profiling of end-stage dilated cardiomyopathy using a human cardiovascular-based cDNA microarray. Am J Pathol. 2002;160:2035-43.

25. Hwang JJ, Allen PD, Tseng GC, Lam CW, Fananapazir L, Dzau VJ, Liew CC. Microarray gene expression profiles in dilated and hypertrophic cardiomyopathic end-stage heart failure. Physiol Genomics. 2002;10:31-44.

26. Grzeskowiak R, Witt H, Drungowski M, Thermann R, Hennig S, Perrot A, et al. Expression profiling of human dilated cardiomyopathy. Cardiovasc Res. 2003;59:400-11.

27. Steenbergen C, Afshari CA, Petranka JG, Collins J, Martin K, Bennett L, et al. Alterations in apoptotic signaling in human idiopathic cardiomyo- pathic hearts in failure. Am J Physiol Heart Circ Physiol. 2003;284: H268-76.

28. Kääb S, Barth AS, Margerie D, Dugas M, Gebauer M, Zwermann L, et al. Global gene expression in human myocardium-oligonucleotide microarray analysis of regional diversity and transcriptional regulation in heart failure. J Mol Med. 2004;82:308-16.

29. Yung CK, Halperin VL, Tomaselli GF, Winslow RL. Gene expression profiles in end-stage human idiopathic dilated cardiomyopathy. Altered expression of apoptotic and cytoskeletal genes. Genomics. 2004;83: 281-97.

30. Kittleson MM, Minhas KM, Irizarry RA, Ye SQ, Edness G, Breton E, et al. Gene expression analysis of ischemic and nonischemic cardiomyopathy: shared and distinct genes in the development of heart failure. Physiol Genomics. 2005;21:299-307.

31. Margulies KB, Matiwala S, Cornejo C, Olsen H, Craven WA, Bednarik D. Mixed messages. Transription patterns in failing and recovering human myocardium. Circ Res. 2005;96:592-9.

32. de Kleijn D, Pasterkamp G. Toll-like receptors in cardiovascular diseases. Cardiovasc Res. 2003;60:58-67.

\section{The Journal of Thoracic and Cardiovascular Surgery Conflict of Interest Policy}

To assure fairness to authors submitting work for consideration in The Journal of Thoracic and Cardiovascular Surgery, a mechanism exists for managing conflicts of interest. The editor and each of the section editors complete a "Conflict of Interest" form that identifies any and all relationships with commercial and other academic entities. When the editor has a potential conflict because of a relationship with another entity or author, the editor appoints an alternate editor from among the section editors or editorial board members who assumes the entire responsibility for final decisions on the manuscript in question. The editor does not read the reviews that are submitted nor engage in discussing the manuscript prior to the final decision. When the conflict of interest involves a section editor, a "guest section editor" is appointed who fills the role normally played by the conflicted section editor. All members of the editorial board and reviewers are asked to indicate any conflict of interest when they agree to review a manuscript. 
TABLE E1. For genes with $q<.17$ (in ascending) the data are presented as the means \pm standard deviations of normalized and transformed expression signals

\begin{tabular}{|c|c|c|c|c|c|c|c|}
\hline \multirow[b]{2}{*}{ Acc.-No } & \multirow[b]{2}{*}{ Gene symbol } & \multicolumn{4}{|c|}{ Mean \pm SD } & \multirow[b]{2}{*}{$p$ value } & \multirow[b]{2}{*}{$q$ value } \\
\hline & & DCM & DCMi & Myocarditis & Pericarditis & & \\
\hline NM_003879 & CFLAR & $0.33 \pm 0.24$ & $0.16 \pm 0.28$ & $0.63 \pm 0.21$ & $0.7 \pm 0.42$ & .000 & .029 \\
\hline NM_000064 & C3 & $0.06 \pm 0.1$ & $0.05 \pm 0.2$ & $0.27 \pm 0.16$ & $0.24 \pm 0.17$ & .000 & .029 \\
\hline NM_001681.2 & SERCA2 & $0.88 \pm 0.41$ & $0.69 \pm 0.42$ & $1.24 \pm 0.22$ & $1.37 \pm 0.36$ & .000 & .029 \\
\hline NM_000062 & SERPING1 & $0.03 \pm 0.18$ & $0.12 \pm 0.21$ & $0.35 \pm 0.22$ & $0.24 \pm 0.22$ & .001 & .075 \\
\hline NM_001734 & C1S & $0.18 \pm 0.17$ & $0.17 \pm 0.17$ & $0.43 \pm 0.22$ & $0.3 \pm 0.04$ & .002 & .113 \\
\hline NM_000153 & GALC & $0.18 \pm 0.21$ & $0.02 \pm 0.21$ & $0.3 \pm 0.17$ & $0.32 \pm 0.22$ & .004 & .152 \\
\hline NM_016562 & TLR7 & $0.01 \pm 0.1$ & $0.0 \pm 0.09$ & $-0.12 \pm 0.09$ & $-0.08 \pm 0.14$ & .005 & .152 \\
\hline NM_013261.1 & PGC-1 & $0.12 \pm 0.09$ & $0.07 \pm 0.1$ & $0.14 \pm 0.11$ & $0.22 \pm 0.09$ & .005 & .152 \\
\hline NM_006573 & TNFSF13B & $-0.23 \pm 0.12$ & $-0.08 \pm 0.09$ & $-0.24 \pm 0.16$ & $-0.22 \pm 0.09$ & .005 & .152 \\
\hline NM_004633 & IL1R2 & $0.03 \pm 0.05$ & $0.1 \pm 0.13$ & $-0.01 \pm 0.06$ & $-0.03 \pm 0.11$ & .006 & .152 \\
\hline NM_005252 & FOS & $0.08 \pm 0.11$ & $0.09 \pm 0.09$ & $0.02 \pm 0.06$ & $-0.07 \pm 0.16$ & .006 & .152 \\
\hline NM_000657 & BCL2.2 & $-0.21 \pm 0.09$ & $-0.13 \pm 0.07$ & $-0.27 \pm 0.09$ & $-0.2 \pm 0.07$ & .007 & .154 \\
\hline NM_080738 & EDARADD & $0.27 \pm 0.19$ & $0.22 \pm 0.19$ & $0.46 \pm 0.14$ & $0.37 \pm 0.17$ & .008 & .154 \\
\hline NM_002521.1 & NPPB & $0.11 \pm 0.6$ & $-0.07 \pm 0.62$ & $-0.26 \pm 0.57$ & $-0.82 \pm 0.65$ & .009 & .154 \\
\hline NM_006301 & MAP3K12 & $-0.07 \pm 0.07$ & $-0.01 \pm 0.07$ & $-0.03 \pm 0.06$ & $-0.18 \pm 0.22$ & .010 & .154 \\
\hline NM_000201.1 & ICAM1 & $-0.08 \pm 0.09$ & $0.02 \pm 0.08$ & $-0.08 \pm 0.1$ & $0.02 \pm 0.09$ & .010 & .154 \\
\hline NM_000874 & IFNAR2 & $0.59 \pm 0.43$ & $0.36 \pm 0.28$ & $0.82 \pm 0.29$ & $0.82 \pm 0.33$ & .010 & .154 \\
\hline NM_003805 & CRADD & $0.02 \pm 0.12$ & $-0.04 \pm 0.12$ & $0.11 \pm 0.09$ & $0.1 \pm 0.13$ & .011 & .154 \\
\hline NM_002745 & MAPK1.1 & $0.22 \pm 0.18$ & $0.15 \pm 0.13$ & $0.32 \pm 0.11$ & $0.33 \pm 0.16$ & .011 & .154 \\
\hline NM_002755 & MAP2K1 & $-0.15 \pm 0.19$ & $-0.03 \pm 0.15$ & $-0.02 \pm 0.28$ & $0.17 \pm 0.41$ & .012 & .154 \\
\hline NM_019846 & CCL28 & $-0.04 \pm 0.11$ & $0.01 \pm 0.08$ & $0.07 \pm 0.16$ & $0 \pm 0.06$ & .012 & .154 \\
\hline NM_006538 & BCL2L11 & $-0.07 \pm 0.06$ & $-0.1 \pm 0.1$ & $0.01 \pm 0.08$ & $-0.02 \pm 0.08$ & .013 & .154 \\
\hline NM_017442 & TLR9 & $0.02 \pm 0.14$ & $0.06 \pm 0.08$ & $0.14 \pm 0.09$ & $0.16 \pm 0.12$ & .013 & .154 \\
\hline NM_001242 & TNFRSF7 & $-0.13 \pm 0.06$ & $-0.06 \pm 0.06$ & $-0.07 \pm 0.09$ & $-0.13 \pm 0.09$ & .013 & .154 \\
\hline NM_000043 & TNFRSF6 & $-0.04 \pm 0.05$ & $-0.01 \pm 0.08$ & $-0.06 \pm 0.1$ & $-0.18 \pm 0.23$ & .014 & .154 \\
\hline NM_000655.2 & SELL & $-0.15 \pm 0.13$ & $0 \pm 0.18$ & $-0.18 \pm 0.1$ & $-0.15 \pm 0.16$ & .014 & .154 \\
\hline NM_000543 & SMPD1 & $0.13 \pm 0.18$ & $0.02 \pm 0.2$ & $0.26 \pm 0.15$ & $0.2 \pm 0.21$ & .017 & .155 \\
\hline NM_002720 & PPP4C & $-0.11 \pm 0.15$ & $0 \pm 0.09$ & $-0.06 \pm 0.11$ & $0.04 \pm 0.04$ & .017 & .155 \\
\hline NM_002188 & IL13 & $0.23 \pm 0.24$ & $0.11 \pm 0.17$ & $0.37 \pm 0.19$ & $0.3 \pm 0.14$ & .018 & .155 \\
\hline NM_021975 & RELA & $-0.14 \pm 0.14$ & $-0.03 \pm 0.07$ & $-0.11 \pm 0.11$ & $-0.02 \pm 0.09$ & .018 & .155 \\
\hline NM_004834 & MAP4K4 & $0.34 \pm 0.29$ & $0.22 \pm 0.25$ & $0.52 \pm 0.19$ & $0.53 \pm 0.31$ & .018 & .155 \\
\hline NM_001350 & DAXX & $-0.01 \pm 0.09$ & $-0.04 \pm 0.12$ & $0.08 \pm 0.09$ & $0.05 \pm 0.14$ & .018 & .155 \\
\hline NM_022740 & HIPK2 & $-0.11 \pm 0.08$ & $-0.03 \pm 0.09$ & $-0.16 \pm 0.1$ & $-0.09 \pm 0.13$ & .019 & .155 \\
\hline NM_004315 & ASAH1 & $-0.09 \pm 0.1$ & $-0.06 \pm 0.13$ & $0.03 \pm 0.1$ & $0.02 \pm 0.16$ & .019 & .156 \\
\hline NM_003376 & VEGF & $0.61 \pm 0.42$ & $0.43 \pm 0.29$ & $0.83 \pm 0.31$ & $0.85 \pm 0.33$ & .020 & .161 \\
\hline NM_004131 & GZMB & $-0.23 \pm 0.11$ & $-0.14 \pm 0.06$ & $-0.22 \pm 0.1$ & $-0.13 \pm 0.1$ & .022 & .162 \\
\hline NM_000256.2 & MYBPC3 & $1.02 \pm 0.43$ & $0.92 \pm 0.3$ & $1.22 \pm 0.21$ & $1.35 \pm 0.17$ & .022 & .162 \\
\hline NM_001225 & CASP4 & $-0.14 \pm 0.11$ & $-0.06 \pm 0.06$ & $-0.03 \pm 0.08$ & $-0.12 \pm 0.12$ & .023 & .162 \\
\hline NM_001824.2 & CKM & $0.79 \pm 0.4$ & $0.78 \pm 0.44$ & $1.1 \pm 0.28$ & $1.2 \pm 0.39$ & .023 & .162 \\
\hline NM_002704 & PPBP & $0.02 \pm 0.15$ & $0.17 \pm 0.45$ & $0.06 \pm 0.16$ & $-0.35 \pm 0.69$ & .025 & .170 \\
\hline NM_032964 & CCL15.2 & $-0.03 \pm 0.1$ & $-0.05 \pm 0.09$ & $0.03 \pm 0.07$ & $0.05 \pm 0.06$ & .025 & .170 \\
\hline NM_000611 & CD59 & $0.09 \pm 0.1$ & $0.04 \pm 0.12$ & $0.17 \pm 0.12$ & $0.17 \pm 0.08$ & .026 & .170 \\
\hline
\end{tabular}

$S D$, Standard deviation; $D C M$, dilated cardiomyopathy; $D C M i$, inflammatory dilated cardiomyopathy. 